\title{
Efficacy of glucocorticoids, conventional and targeted synthetic disease-modifying antirheumatic drugs: a systematic literature review informing the 2016 update of The EULAR recommendations for the management of rheumatoid arthritis
}

\author{
Katerina Chatzidionysiou, ${ }^{1}$ Sharzad Emamikia, ${ }^{1}$ Jackie Nam, ${ }^{2}$ Sofia Ramiro, ${ }^{3}$ \\ Josef Smolen, ${ }^{4}$ Désirée van der Heijde, ${ }^{3}$ Maxime Dougados, ${ }^{5}$ Johannes Bijlsma, ${ }^{6}$ \\ Gerd Burmester, ${ }^{7}$ Marieke Scholte, ${ }^{8,9}$ Ronald van Vollenhoven, ${ }^{1,10}$ Robert Landewé ${ }^{10}$
}

Handling editor Dr. Francis Berenbaum

- Additional material is published online only. To view please visit the journal online (http://dx.doi.org/10.1136/ annrheumdis-2016-210711)

For numbered affiliations see end of article.

Correspondence to Dr Katerina Chatzidionysiou, Unit for Clinical Therapy Research, Inflammatory Diseases (ClinTRID),

Rheumatology Department, Karolinska University Hospital, The Karolinska Institute, Stockholm 171 76, Sweden; aikaterini.chatzidionysiou@ karolinska.se

Received 21 October 2016 Revised 26 January 2017 Accepted 28 February 2017 Published Online First 29 March 2017

\section{Linked}

- http://dx.doi.org/10.1136/ annrheumdis-2016-210715

- http://dx.doi.org/10.1136/ annrheumdis-2016-211005

\section{CrossMark}

To cite: Chatzidionysiou K,

Emamikia S, Nam J,

et al. Ann Rheum Dis

2017:76:1102-1107.

\section{ABSTRACT}

Objectives To perform a systematic literature review (SLR) informing the 2016 update of the

recommendations for the management of rheumatoid arthritis (RA).

Methods An SLR for the period between 2013 and 2016 was undertaken to assess the efficacy of glucocorticoids (GCs), conventional synthetic diseasemodifying antirheumatic drugs (csDMARDs) and targeted synthetic DMARDs (tsDMARDs) (tofacitinib and

baricitinib) in randomised clinical trials.

Results For GCs, four studies were included in the SLR. Patients without poor prognostic factors experienced benefit when GCs were added to methotrexate (MTX). Lower doses of GCs were similar to higher doses. For cSDMARDs, two new studies comparing MTX

monotherapy with combination csDMARD were included in the SLR. In the tREACH trial at the end of 12 months no difference between the groups in disease activity, functional ability and radiographic progression was seen, using principles of tight control (treat-to-target). In the CareRA trial, combination therapy with csDMARDs was not superior to MTX monotherapy and monotherapy was better tolerated.

For tsDMARDs, tofacitinib and baricitinib were shown to be more effective than placebo (MTX) in different patient populations.

Conclusions Addition of GCs to csDMARD therapy may be beneficial but the benefits should be balanced against the risk of toxicity. Under tight control conditions MTX monotherapy is not less effective than combination csDMARDs, but better tolerated. Tofacitinib and baricitinib are efficacious in patients with RA, including those with refractory disease.

\section{INTRODUCTION}

The landscape of rheumatoid arthritis (RA) treatment has unquestionably changed dramatically during the last decade. The development and introduction to daily clinical practice of disease modifying antirheumatic drugs (DMARDs) as well as earlier diagnosis and treatment, and well defined goals of treatment, have contributed to this treatment revolution. Despite this progress, there are still unmet needs, and a better application of the currently available treatments as well as better treatment strategies are needed. Practical recommendations based on the existing evidence are appropriate tools for the rheumatologists. In 2013 a European League Against Rheumatism (EULAR) task force has revised the previous recommendations on RA treatment. ${ }^{1}$ A revision of the 2013 recommendations was now undertaken.

The aim of this review was to inform the new EULAR recommendations ${ }^{2}$ on the management of RA on efficacy of glucocorticoids (GCs), conventional synthetic DMARDs (csDMARDs) and two targeted synthetic DMARDs (tsDMARDs), tofacitinib and baricitinib based on new evidence accrued since $2013 .^{3}$ The results of this and two other systematic literature reviews (SLRs) ${ }^{4}$ provided the task force with the current state of evidence.

\section{METHODS}

An SLR using MEDLINE, EMBASE and the Cochrane CENTRAL library was performed from January 2013 until February 2016, based on a prespecified PICOS statement: $\mathrm{P}=$ population, $\mathrm{I}=$ interventions,$\quad \mathrm{C}=$ comparators, $\mathrm{O}=$ outcomes and $\mathrm{S}=$ study design. The population was 'adult RA patients'; the intervention was (1) GCs, (2) csDMARDs (methotrexate (MTX), leflunomide, sulfasalazine, hydroxychloroquine, intramuscular gold, auranofin, azathioprine, ciclosporine, minocycline, D-penicillamine, cyclophosphamide, chlorambucil, mycophenolate, tacrolimus), (as monotherapy or combination therapy) and (3) tsDMARDs (tofacitinib and baricitinib); the comparator was patients not receiving the abovementioned treatments; the outcome pertained to efficacy on disease activity, function, patient reported outcomes (PROs) and structural damage; and the study design always was 'randomised controlled trials' (RCTs). Risk of bias (RoB) was assessed using the Cochrane RoB assessment tool (Cochrane Handbook for Systematic Reviews of Interventions V.5.1.0 March 2011 (cited September 2016); available from: http://handbook.cochrane. org/). ORs for dichotomous measures were 
determined to assess the magnitude of treatment effect. The DerSimonian and Laird random-effects model was used to pool the data when possible, allowing for both within-study and between-study variations. Statistical heterogeneity among studies was evaluated using the $\mathrm{I}^{2}$ statistic and $\chi^{2}$ test where a $\mathrm{p}$ value $<0.10$ was considered to be statistically significant. A value of above $50 \%$ for $\mathrm{I}^{2}$ was considered to be high. Details about the search and the studies included can be found in the online supplementary material. The selected group of patients included in RCTs as well as the relatively short duration of RCTs, makes addressing long-term safety of drugs in RCTs difficult. For this reason, safety aspects of GCs and csDMARDs were addressed in a separate SLR based on observational studies coming from registries. ${ }^{5}$ Some safety issues regarding tsDMARDs will be discussed here, since real life data of tsDMARDs are still lacking.

\section{RESULTS}

\section{Efficacy of addition of GCs to csDMARDs}

Of 348 hits, 4 studies were included in the analysis (table 1). The selection of articles is shown in online supplementary figure S1. A small study by Menon $e t a l^{6}$ showed greater efficacy of a combination of csDMARDs with intra-articular GCs than with csDMARDs alone in patients with RA with less than 2 years disease duration, but this was an open label study with high RoB. In the CareRA trial patients with early RA, but without poor prognostic factors, benefited from the addition of GCs (COBRA-slim) to MTX with no differences in safety observed. ${ }^{7}$ The primary end point of this study was not met, since the percentage of patients achieving remission at week 16 was only numerically but not significantly higher in the GC group $(65.1 \%$ vs $46.8 \%, \mathrm{p}=0.08)$. However, this substudy analysis did not have sufficient statistical power and had a high RoB, primarily due to lack of blinding.

A non-inferiority trial compared two different GC strategies; the COBRA-light strategy (prednisolone at $30 \mathrm{mg} /$ day, tapered to $7.5 \mathrm{mg} /$ day in 9 weeks) in combination with MTX; and the COBRA strategy, using prednisolone at $60 \mathrm{mg} /$ day (tapered to $7.5 \mathrm{mg} /$ day in 6 weeks) in combination with both MTX and sulfasalazine. The lower dose of GCs was efficacious in suppressing clinical disease activity and improving functional ability, but non-inferiority could not be claimed formally. ${ }^{8}$ The degree of radiographic progression was similar in the two groups (COBRA and COBRA-light). However, this study also had a high RoB (open design), and no comparison with application of conventional GCs was performed.

In a double-blind RCT with patients with established RA, low-dose prednisone with modified release ('chronotherapy') added to existing DMARD treatment in patients with active disease had a significant effect on disease activity and health-related quality of life compared with placebo. ${ }^{10}$

A pooled analysis could not be performed because of significant heterogeneity of the studies regarding designs, patient populations, doses and routes of administration of GCs, and outcome measures. The results of the newer RCTs are in accordance with the previously formulated standpoint that GC when added to csDMARD therapy may have beneficial effects. Safety aspects, as addressed in a separate SLR, have to be taken into consideration. ${ }^{5}$ Level of evidence (LOE): $1 \mathrm{a}$.

\section{Efficacy of csDMARDs and csDMARD combinations}

In total 518 studies were screened. The selection of articles is shown in online supplementary figure S2. Only two new studies comparing MTX monotherapy with MTX in combination with another csDMARD without differences in GC usage were

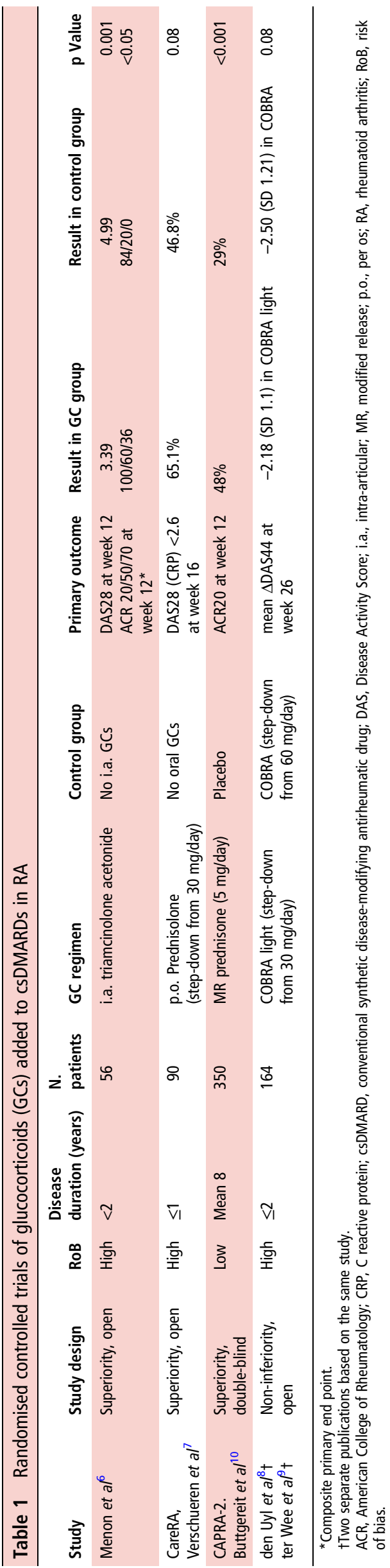


included in the SLR. In the tREACH trial, that applied tight control principles, at 12 months, disease activity, functional ability and radiographic progression were similar in the two groups who received csDMARD combination therapy (MTX, sulfasalazine and hydroxychloroquine) with either oral GCs or intramuscular GCs and the group that received MTX monotherapy (see online supplementary table S1). GCs were given either intramuscularly (methylprednisolone $120 \mathrm{mg}$ or triamcinolone $80 \mathrm{mg}$ ) or in an oral tapering scheme (weeks 1-4: $15 \mathrm{mg} / \mathrm{day}$, weeks 5-6: $10 \mathrm{mg} /$ day, weeks 7-8: $5 \mathrm{mg} /$ day and weeks 9-10: $2.5 \mathrm{mg} /$ day). In addition, a higher number of medication adjustments due to adverse events (AEs) were applied in the combination group. ${ }^{11}{ }^{12}$ Interestingly, for the two groups on combination therapy, intramuscular and oral GCs were similarly effective as modes of bridging therapy.

In the CareRA trial (in a different subpopulation than the one described above in a different part of the CareRA trial) patients with early RA and risk factors for more aggressive disease did not benefit from combination of MTX with other csDMARDs in comparison to MTX monotherapy (both combined with GCs) (see online supplementary table S1). In these arms GCs were dosed orally using a weekly step-down scheme (30-2012.5-10-7.5-5 mg prednisone). Monotherapy with MTX was better tolerated. ${ }^{13}$ The CareRA trial has a high RoB (open label).

The results of the newer RCTs are in accordance with the previously formulated standpoint that combination of csDMARDs is not better than monotherapy with MTX. The need for more optimal use of csDMARDs, particularly regarding the dose of csDMARDs, however, is obvious. One double-blind RCT failed to show differences between two starting doses of MTX, namely $7.5 \mathrm{mg}$ and $15 \mathrm{mg}$ weekly. ${ }^{14}$ In the CONCERTO trial initiating adalimumab + MTX combination therapy, the efficacy of $10 \mathrm{mg} /$ week and $20 \mathrm{mg} /$ week MTX was not statistically different in patients with early RA. ${ }^{15}$ One study compared a loading dose of leflunomide ( $100 \mathrm{mg} \times 1$ for 3 days) with a fixed dose of $20 \mathrm{mg}$ daily and did not show differences in efficacy but a better safety profile for the fixed dose. ${ }^{16} \mathrm{~A}$ weekly dose of $50 \mathrm{mg}$ leflunomide showed similar benefits to a daily dose of $10 \mathrm{mg}$ leflunomide for the treatment of mild-to-moderate early RA. ${ }^{17}$ The latter however was an open superiority study with a high RoB and $10 \mathrm{mg}$ leflunomide daily is considered a suboptimal dose.

\section{Efficacy of tsDMARDs (tofacitinib and baricitinib)}

From the 134 hits on tofacitinib 9 were identified as RCTs (table 2) ${ }^{18-26}$ Efficacy of tofacitinib, both as monotherapy and in combination with MTX, was formally proven in different patient populations (MTX-naïve, csDMARD and biological DMARD (bDMARD) inadequate responders) compared with placebo (background MTX). For baricitinib the literature search yielded eight new RCTs (two of them had PROs as main study outcomes) (table 3). ${ }^{27-34}$ Similar clinical efficacy of baricitinib in monotherapy and in combination with MTX has been suggested, but only the combination (baricitinib + MTX) significantly inhibited radiographic progression. ${ }^{29}$ In the MTX-IR (inadequate responder) RA-BEAM study, comparing adalimumab+MTX versus baricitinib+MTX versus placebo+MTX, showed small but significantly lower responses for adalimumab + MTX versus baricitinib + MTX, but both were higher than placebo+MTX (Disease Activity Score 28-C reactive protein $<2.619 \%$ vs $24 \%$ vs $4 \%$ ) at week 12 . $^{30}$

Importantly, baricitinib has now shown efficacy in a refractory RA population after failure of both antitumour necrosis factor
(anti-TNF) and non-anti-TNF bDMARDs. ${ }^{16}$ All studies had low RoB. The selection of articles for tofacitinib and baricitinib is shown in online supplementary figures S3 and S4, respectively.

No meta-analysis could be performed due to the heterogeneity between the studies. The most commonly found laboratory abnormalities with tofacitinib were mild decreases in neutrophil and lymphocyte counts and mild increases in aminotransferase and creatinine levels, while baricitinib was associated with reductions in haemoglobin levels. The relative risks for serious AEs with tofacitinib and baricitinib compared with placebo were 0.8 (95\% CI 0.5 to 1.3 ) and 1.0 (95\% CI 0.6 to 1.7$)$, respectively. However, a significantly increased risk of herpes infection was seen $(\mathrm{RR}=3.1,95 \% \mathrm{CI} 1.1$ to 8.5$)$ with tofacitinib.

The results of the newer RCTs are in accordance with the previously formulated standpoint that the tsDMARDs (tofacitinib and baricitinib) are effective and safe in the short term. (LOE: 1A)

\section{DISCUSSION}

Overall, the results of this review confirmed the previous SLR and expanded the overall insights. Although the evidence on efficacy of short-term GCs when added to csDMARDs is robust and undisputed, there are still concerns regarding long-term safety (such as infections, diabetes, osteoporosis, and gastrointestinal and cardiovascular events). Preliminary long-term results of the CAMERA II trial showed a low occurrence of AEs but suggested for the first time an increased cardiovascular risk for the patients with early RA treated with $10 \mathrm{mg} /$ day prednisone for at least 2 years. ${ }^{35}$ These results are still unpublished (abstract in American College of Rheumatology 2015). A separate SLR focusing on the safety of GCs has been performed in order to inform the task force and enable the formation of the recommendations. ${ }^{5}$ GC safety aspects have also been addressed in a separate paper prior EULAR activity. ${ }^{36}$ Clear consensus regarding the dose and tapering of GCs is still lacking. New data have suggested that short-term lower doses of GCs (starting at $30 \mathrm{mg}$ prednisone per day with rapid tapering), as in the COBRA-light regimen, might be a feasible alternative to the higher doses (starting at $60 \mathrm{mg} /$ day) as in the COBRA regimen, although formal non-inferiority was not proven. In fact, this trial did not fulfil the inclusion criteria for the SLR, since there was no comparator group (group without GCs according to the PICO). However, we decided to include it in the SLR since the question posed is highly clinically relevant.

Interestingly, the tREACH trial has suggested that the efficacy of oral GCs as bridging treatments was not superior to intramuscular GCs. Two new studies were published regarding chronotherapy and intra-articular GC therapy, thus answering one of the research questions posed in 2013. The latter however was a high RoB study.

Regarding the choice of csDMARD combination therapy over monotherapy, again-and in contradiction with the perception of many clinicians-we could not substantiate clear evidence in favour of combination therapy with csDMARDs. Neither the 1-year results of the tREACH, nor those of the CARERA study, showed clear evidence that MTX monotherapy is inferior to combination therapy with csDMARDs when used in combination with GCs and when a tight treat-to-target approach is employed. Importantly, monotherapy was generally better tolerated than combination therapy in these studies. Generally, the complexity of the design of pragmatic trials and certain methodological issues, such as high dropout rates and change of primary end point, make the interpretation of the results challenging. 


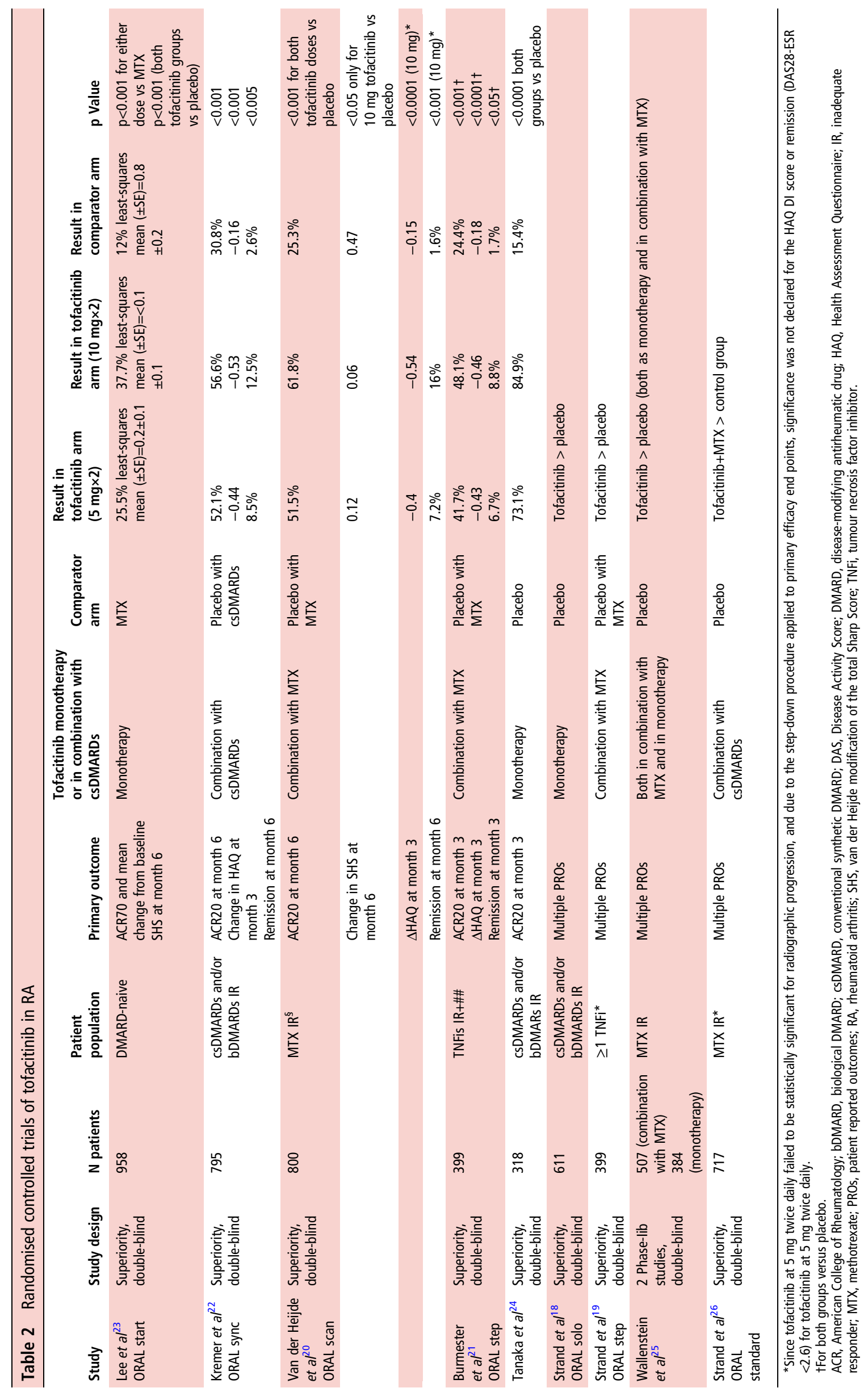




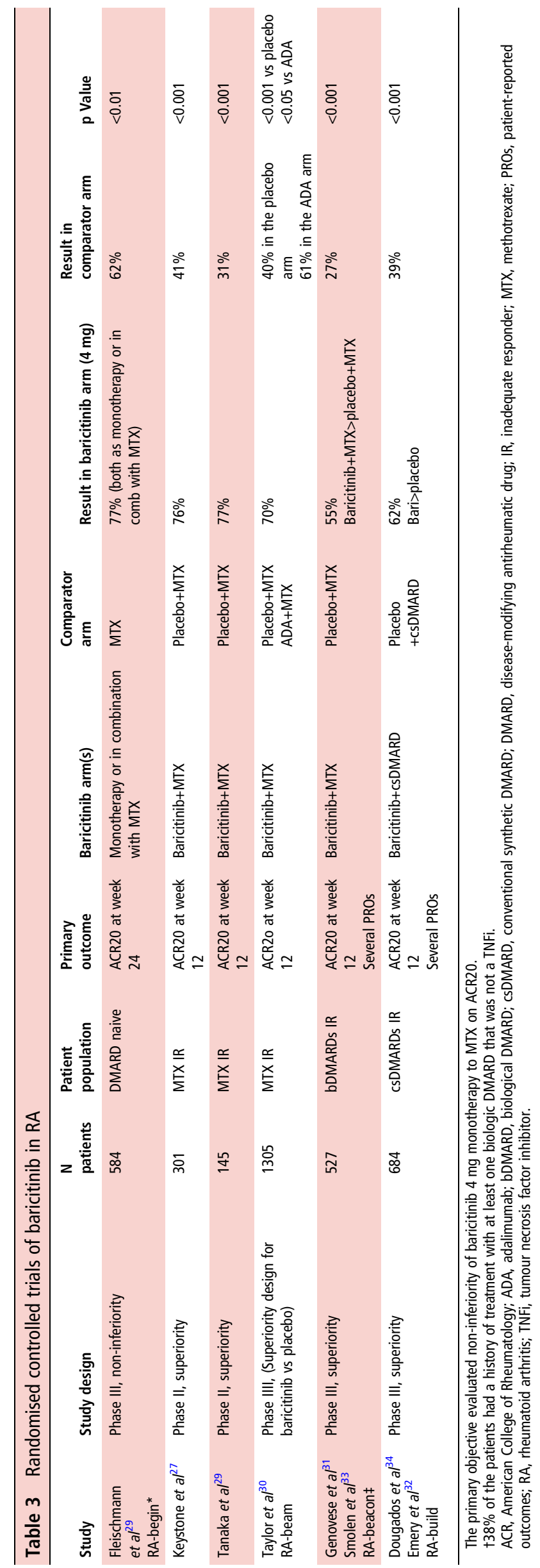

There is a clear need for studies addressing the optimal use of csDMARDs. No new studies fulfilling the inclusion criteria regarding dose and route of administration of MTX were identified. A previous SLR by Visser and van der $\mathrm{Heijde}^{37}$ had addressed this issue.

Tofacitinib is the first JAK inhibitor approved for the treatment of RA in many countries and baricitinib is under regulatory evaluation. This SLR confirmed that tofacitinib has beneficial effects on disease activity, physical function, radiographic progression and PROs, both in patients with early RA who are DMARD-naive and in patients with established disease who have failed csDMARDs and/or bDMARDs. Baricitinib was found to be effective in MTX-naïve patients and also after failure of drugs with multiple modes of action. Data on longterm safety of this new class of DMARDs from real life observational studies are needed. Until then, rheumatologists are advised to take into account safety data obtained through RCTs and follow the labels of each drug, including AEs and lab monitoring.

\section{Author affiliations}

${ }^{1}$ Karolinska Institute, Stockholm, Sweden

${ }^{2}$ Leeds Institute of Rheumatic and Musculoskeletal Medicine, University of Leeds, Leeds, UK

${ }^{3}$ LUMC, Leiden, The Netherlands

${ }^{4}$ Medical University of Vienna, Vienna, Austria

${ }^{5}$ Paris Descartes University, Paris, France

${ }^{6}$ Utrecht University Medical Center, Utrecht, The Netherlands

${ }^{7}$ Charité University Hospital, Berlin, Germany

${ }^{8}$ Department of Psychology, Health and Technology, University of Twente, Enschede, The Netherlands

${ }^{9}$ EULAR Standing Committee of People with Arthritis/Rheumatism in Europe

${ }^{10}$ Amsterdam Rheumatology and Immunology Center, Amsterdam, The Netherlands

Contributors All authors contributed and finally approved the current manuscript. Competing interests None declared.

Provenance and peer review Not commissioned; externally peer reviewed.

\section{REFERENCES}

1 Smolen JS, Landewé $\mathrm{R}$, Breedveld FC, et al. EULAR recommendations for the management of rheumatoid arthritis with synthetic and biological disease-modifying antirheumatic drugs: 2013 update. Ann Rheum Dis 2014;73:492-509.

2 Smolen JS, Landewé R, Breedveld FC, et al. EULAR recommendations for the management of rheumatoid arthritis with synthetic and biological disease modifying antirheumatic drugs - 2016 update. Ann Rheum Dis 2013;-m, submitted 2016.

3 Gaujoux-Viala C, Nam J, Ramiro S, et al. Efficacy of conventional synthetic disease-modifying antirheumatic drugs, glucocorticoids and tofacitinib: a systematic literature review informing the 2013 update of the EULAR recommendations for management of rheumatoid arthritis. Ann Rheum Dis 2014;73:510-15.

4 Nam J, Takase-Minegishi K, Ramiro S, et al. Efficacy of biological DMARDs-a systematic literature review informing the 2016 update of the EULAR recommendations for management of rheumatoid arthritis. Ann Rheum Dis 2016; - nn, submitted.

5 Ramiro S, Gaujoux-Viala C, Nam J, et al. Safety of synthetic and biological DMARDs - a systematic literature review informing the 2016 update of the EULAR recommendations for management of rheumatoid arthritis. Ann Rheum Dis mSubmitted 2016.

6 Menon N, Kothari SY, Gogna A, et al. Comparison of intra-articular glucocorticoid injections with DMARDs versus DMARDs alone in rheumatoid arthritis. J Assoc Physicians India 2014;62:673-6.

7 Verschueren P, De Cock D, Corluy L, et al. Patients lacking classical poor prognostic markers might also benefit from a step-down glucocorticoid bridging scheme in early rheumatoid arthritis: week 16 results from the randomized multicenter CareRA trial. Arthritis Res Ther 2015;17:97.

8 den Uyl D, ter Wee M, Boers $\mathrm{M}$, et al. A non-inferiority trial of an attenuated combination strategy ('COBRA-light') compared to the original COBRA strategy: clinical results after 26 weeks. Ann Rheum Dis 2014;73:1071-8.

9 ter Wee MM, den Uyl D, Boers $M$, et al. Intensive combination treatment regimens, including prednisolone, are effective in treating patients with early rheumatoid arthritis regardless of additional etanercept: 1-year results of the COBRA-light open-label, randomised, non-inferiority trial. Ann Rheum Dis 2015;74:1233-40. 
10 Buttgereit F, Mehta D, Kirwan J, et al. Low-dose prednisone chronotherapy for rheumatoid arthritis: a randomised clinical trial (CAPRA-2). Ann Rheum Dis 2013;72:204-10.

11 de Jong PH, Hazes JM, Barendregt PJ, et al. Induction therapy with a combination of DMARDs is better than methotrexate monotherapy: first results of the TREACH trial. Ann Rheum Dis 2013;72:72-8.

12 de Jong PH, Hazes JM, Han HK, et al. Randomised comparison of initial triple DMARD therapy with methotrexate monotherapy in combination with low-dose glucocorticoid bridging therapy; 1-year data of the tREACH trial. Ann Rheum Dis 2014:73:1331-9.

13 Verschueren $\mathrm{P}$, De Cock D, Corluy $\mathrm{L}$, et al. Methotrexate in combination with other DMARDs is not superior to methotrexate alone for remission induction with moderate-to-high-dose glucocorticoid bridging in early rheumatoid arthritis after 16 weeks of treatment: the CareRA trial. Ann Rheum Dis 2015:74:27-34.

14 Dhir V, Singla M, Gupta N, et al. Randomized controlled trial comparing 2 different starting doses of methotrexate in rheumatoid arthritis. Clin Ther 2014;36:1005-15.

15 Burmester GR, Kivitz AJ, Kupper $H$, et al. Efficacy and safety of ascending methotrexate dose in combination with adalimumab: the randomised CONCERTO trial. Ann Rheum Dis 2015:74:1037-44.

16 Cutolo M, Bolosiu H, Perdriset G. Efficacy and safety of leflunomide in DMARD-naive patients with early rheumatoid arthritis: comparison of a loading and a fixed-dose regimen. Rheumatology 2013;52:1132-40.

17 Ren LM, Li R, Chen LN, et al. Efficacy and safety of weekly leflunomide for the treatment of early rheumatoid arthritis: a randomized, multi-center study. Int J Rheum Dis 2016;19:651-7.

18 Strand V, Kremer J, Wallenstein G, et al. Effects of tofacitinib monotherapy on patient-reported outcomes in a randomized phase 3 study of patients with active rheumatoid arthritis and inadequate responses to DMARDs. Arthritis Res Ther 2015; 17:307.

19 Strand V, Burmester GR, Zerbini CA, et al. Tofacitinib with methotrexate in third-line treatment of patients with active rheumatoid arthritis: patient-reported outcomes from a phase III trial. Arthritis Care Res (Hoboken) 2015;67:475-83.

20 van der Heijde D, Tanaka Y, Fleischmann R, et al. Tofacitinib (CP-690,550) in patients with rheumatoid arthritis receiving methotrexate: twelve-month data from a twenty-four-month phase III randomized radiographic study. Arthritis Rheum 2013;65:559-70.

21 Burmester GR, Blanco R, Charles-Schoeman C, et al. Tofacitinib (CP-690,550) in combination with methotrexate in patients with active rheumatoid arthritis with an inadequate response to tumour necrosis factor inhibitors: a randomised phase 3 trial. Lancet 2013:381:451-60.

22 Kremer J, Li ZG, Hall S, et al. Tofacitinib in combination with nonbiologic disease-modifying antirheumatic drugs in patients with active rheumatoid arthritis a randomized trial. Annals of Internal Medicine 2013;159:253-61.

23 Lee EB, Fleischmann $\mathrm{R}$, Hall $\mathrm{S}$, et al. Tofacitinib versus methotrexate in rheumatoid arthritis. N Engl J Med 2014;370:2377-86.
24 Tanaka Y, Takeuchi T, Yamanaka H, et al. Efficacy and safety of tofacitinib as monotherapy in Japanese patients with active rheumatoid arthritis: a 12-week, randomized, phase 2 study. Mod Rheumatol 2015;25:514-21.

25 Wallenstein GV, Kanik KS, Wilkinson B, et al. Effects of the oral Janus kinase inhibitor tofacitinib on patient-reported outcomes in patients with active rheumatoid arthritis: results of two Phase 2 randomised controlled trials. Clin Exp Rheumatol 2016;34:430-42.

26 Strand V, van Vollenhoven RF, Lee EB, et al. Tofacitinib or adalimumab versus placebo: patient-reported outcomes from a phase 3 study of active rheumatoid arthritis. Rheumatology 2016;55:1031-41.

27 Keystone EC, Taylor PC, Drescher E, et al. Safety and efficacy of baricitinib at 24 weeks in patients with rheumatoid arthritis who have had an inadequate response to methotrexate. Ann Rheum Dis 2015;74:333-40.

28 Tanaka Y, Emoto K, Cai Z, et al. Efficacy and safety of baricitinib in Japanese patients with active rheumatoid arthritis receiving background methotrexate therapy: a 12-week, double-blind, randomized placebo-controlled study. J Rheumatol 2016:43:504-11.

29 Fleischmann R, Takeuchi T, Schlichting DE, et al. Baricitinib, Methotrexate, or Baricitinib Plus Methotrexate in Patients with Early Rheumatoid Arthritis Who Had Received Limited or No Treatment with Disease-Modifying Anti-Rheumatic Drugs (DMARDs): Phase 3 Trial Results. Arthritis Rheumatol 2015;67:506-17.

30 Taylor PC, Keystone EC, van der Heijde D, et al. Baricitinib Versus Placebo or Adalimumab in Patients with Active Rheumatoid Arthritis (RA) and an Inadequate Response to Background Methotrexate Therapy: Results of a Phase 3 Study. Ann Rheum Dis 2015;67:14-16.

31 Genovese MC, Kremer J, Zamani O, et al. Baricitinib in patients with refractory rheumatoid arthritis. N Engl J Med 2016;374:1243-52.

32 Emery P, Gaich CL, DeLozier AM, et al. Patient-Reported Outcomes from a Phase 3 Study of Baricitinib in Patients with Rheumatoid Arthritis with Inadequate Response to Conventional Synthetic Disease-Modifying Antirheumatic Drugs. Arthritis Rheumatol 2015;67.

33 Smolen JS, Kremer J, Gaich C, et al. Patient-Reported Outcomes from a Phase 3 Study of Baricitinib in Patients with Rheumatoid Arthritis (RA) and an Inadequate Response to Tumor Necrosis Factor Inhibitor. Ann Rheum Dis 2015;74(Suppl 2):785-6.

34 Dougados $M$, van der Heijde $D$, Chen $Y C$, et al. Baricitinib in patients with inadequate response or intolerance to conventional synthetic DMARDs: results from the RA-BUILD study. Ann Rheum Dis 2017;76:88-95.

35 de Hair M, liff N, Jacobs J, et al. Long-Term Adverse Events after Daily Concomitant Treatment with $10 \mathrm{mg}$ Prednisone in the 2-Year Computer Assisted Management in Early Rheumatoid Arthritis Trial-II. Arthritis Rheumatol 2015;67.

36 Hoes JN, Jacobs JW, Boers $M$, et al. EULAR evidence-based recommendations on the management of systemic glucocorticoid therapy in rheumatic diseases. Ann Rheum Dis 2007;66:1560-7.

37 Visser K, van der Heijde D. Optimal dosage and route of administration of methotrexate in rheumatoid arthritis: a systematic review of the literature. Ann Rheum Dis 2009;68:1094-9. 